\title{
Referencias de orientación espacial en las App de Anatomía 3D.
}

\section{Spatial Orientation References in 3D Anatomy Apps.}

\author{
Verónica D'Angelo ${ }^{1,2, *}$ \\ ${ }^{1}$ Universidad Abierta Interamericana; ${ }^{2}$ IRICE (CONICET/ UNR) Instituto Rosario de Investigaciones en \\ Ciencias de la Educación; ORCID ID: 0000-0002-3170-4313 \\ * Correspondencia: Veronica.DAngelo@uai.edu.ar
}

Recibido: 26-3-21; Aceptado: 24-6-21; Publicado: 28-6-21

\begin{abstract}
Resumen: En el presente trabajo de investigación se evaluó la percepción de aplicaciones de anatomía 3D en relación a una estrategia de enseñanza basada en fundamentos cognitivos de la manipulación de imágenes digitales. Dos grupos de estudiantes ingresantes a la carrera de medicina recibieron entrenamiento en el uso de aplicaciones de software de anatomía en 3D, pero sólo uno de los grupos recibió información adicional sobre las limitaciones de la memoria en la retención de imágenes tridimensionales y estrategias para el uso apropiado de los controles de rotación que evitan la sobrecarga cognitiva. La percepción se evaluó con un modelo de aceptación de tecnología (TAM) y se constató una diferencia significativa entre ambos grupos a favor del grupo entrenado. Inferimos que la incorporación de aplicaciones de anatomía 3D en dispositivos tradicionales de pantalla 2D, tendrá mayor aceptación (es decir, probabilidad de uso futuro) cuando esté acompañada de una estrategia de autoregulación para evitar los efectos de desorientación que producen las rotaciones no controladas características del uso autodidacta.
\end{abstract}

Palabras clave: psicología cognitiva; medicina; anatomía 3D; orientación espacial; inteligencia espacial

\begin{abstract}
In this research work the perception of 3D anatomy applications was evaluated in relation to a teaching strategy based on cognitive foundations of digital image manipulation. Two groups of students entering the medical career received training in the use of 3D anatomy software applications, only one of the groups received additional information on memory limitations in the retention of three-dimensional images and strategies for appropriate use of rotation controls that avoid cognitive overload. Perception was evaluated with a technology acceptance model (TAM) and a significant difference was found between both groups in favor of the trained group. We infer that the incorporation of 3D anatomy applications in traditional 2D screen devices will have greater acceptance (that is, probability of future use) when it is accompanied by a self-regulation strategy to avoid the disorientation effects produced by the characteristic uncontrolled rotations in self-taught use.
\end{abstract}

Keywords: cognitive psychology; medicine; 3D anatomy; Spatial Orientation; spatial intelligence

\section{Introducción.}

Las investigaciones educativas sobre inclusión de tecnologías digitales en el ámbito latinoamericano se han caracterizado por una adhesión generalizada a la metáfora nativos digitales (1) y por la ausencia de los enfoques cognitivos producto de su difícil recepción en las facultades de psicología (2-3). El resultado es que los medios digitales han sido incorporados de manera ingenua, sin indagación sobre sus limitaciones metacognitivas. Como no existen grandes diferencias entre las interfaces de aplicaciones en lo que respecta a la operatividad, los estudiantes suelen comenzar la exploración de un nuevo software de manera espontánea y con poca tolerancia al error. Esperan resultados claros, tangibles y 
rápidos. Cuando ciertas funciones refieren a conceptos complejos o desconocidos, suelen frustrarse rápidamente y percibir la aplicación de software de una manera negativa. En tales casos, una guía adecuada es fundamental para evitar la decepción y el desaprovechamiento de recursos útiles.

En el campo de la medicina en particular son populares las aplicaciones 3D para el aprendizaje de anatomía humana (4) (sobre origen y ventajas de las aplicaciones 3D, ver puntos 1 y 2 del Anexo). Si bien está claro que las App contribuyen a facilitar la exploración, no se ha comprobado que mejoren específicamente el aprendizaje de conceptos de anatomía. De hecho, en los últimos años, se ha constatado repetidamente que el conocimiento de los estudiantes sobre anatomía es insuficiente (5-8). El aprovechamiento de las aplicaciones 3D interactivas depende de factores críticos como la inteligencia espacial de los usuarios (18) y el empleo de planos canónicos (sobre el procesamiento cognitivo de la información visual, ver Anexo, 3). Para un alumno con baja habilidad espacial el 3D puede ser contraproducente (Anexo, 4). Hace más de dos décadas comenzaron a investigarse las desventajas, para algunos alumnos, de utilizar aplicaciones en 3D (9-10) así como las implicaciones para el desarrollo del software (11-17). Para reducir las consecuencias de la sobre carga cognitiva de los modelos 3D, los usuarios deberían estar al tanto de las referencias de orientación espacial incluidas en el software, o guiados por un docente que conozca la importancia de dichas orientaciones (Ver Anexo, $5)$.

A pesar del tiempo transcurrido, si bien se han incorporado ayudas en las App, especialmente las referencias de orientación espacial, este tema no ha tenido gran impacto en la educación médica local. En una exploración previa al presente estudio, se encuestó a 45 docentes de la carrera de medicina en dos universidades de la ciudad Rosario, Argentina, con las siguientes preguntas:

1. ¿Cree que los estudiantes de medicina necesitan ser orientados por un docente para utilizar aplicaciones de anatomía $3 D$ en su celular o computadora?

2. ¿Cree que los estudiantes de medicina necesitan inteligencia viso espacial?

3. ¿Conoce las referencias de orientación espacial en las App de anatomía 3D?

Todos los docentes encuestados conocían la existencia de las aplicaciones 3D y el uso que hacían los alumnos, aunque no se registró si las utilizaban como material didáctico en sus clases. El 84,4\% de los docentes respondió a favor del autodidactismo digital de los alumnos, es decir, que los estudiantes no necesitan ser orientados por un docente para usar aplicaciones 3D. Sólo el 6,7\% cree que los estudiantes necesitan inteligencia visoespacial, 17.8 creen que no la necesitan, y $75,6 \%$ no lo saben (es posible que desconozcan el concepto). Sólo un 2,2\% dijo conocer las referencias de orientación espacial mientras que el $97,8 \%$ las desconoce.

\subsection{Referencias de orientación espacial}

Debido a las dificultades de los ratones 2D tradicionales para manipular representaciones 3D (Ver Anexo, 5.1) y para evitar la desorientación producida por las rotaciones libres en la pantalla, varias aplicaciones de anatomía incluyen referencias a la rotación espacial. A continuación, describiremos las referencias incluidas en Visible Body. En el punto 5.2. del Anexo pueden consultarse otras aplicaciones de propósito educativo.

\subsubsection{Visible Body.}

Esta aplicación contiene un control de rotación denominado "Palanca de mando" (Figura 1) que se activa desde el menú Configuración. 


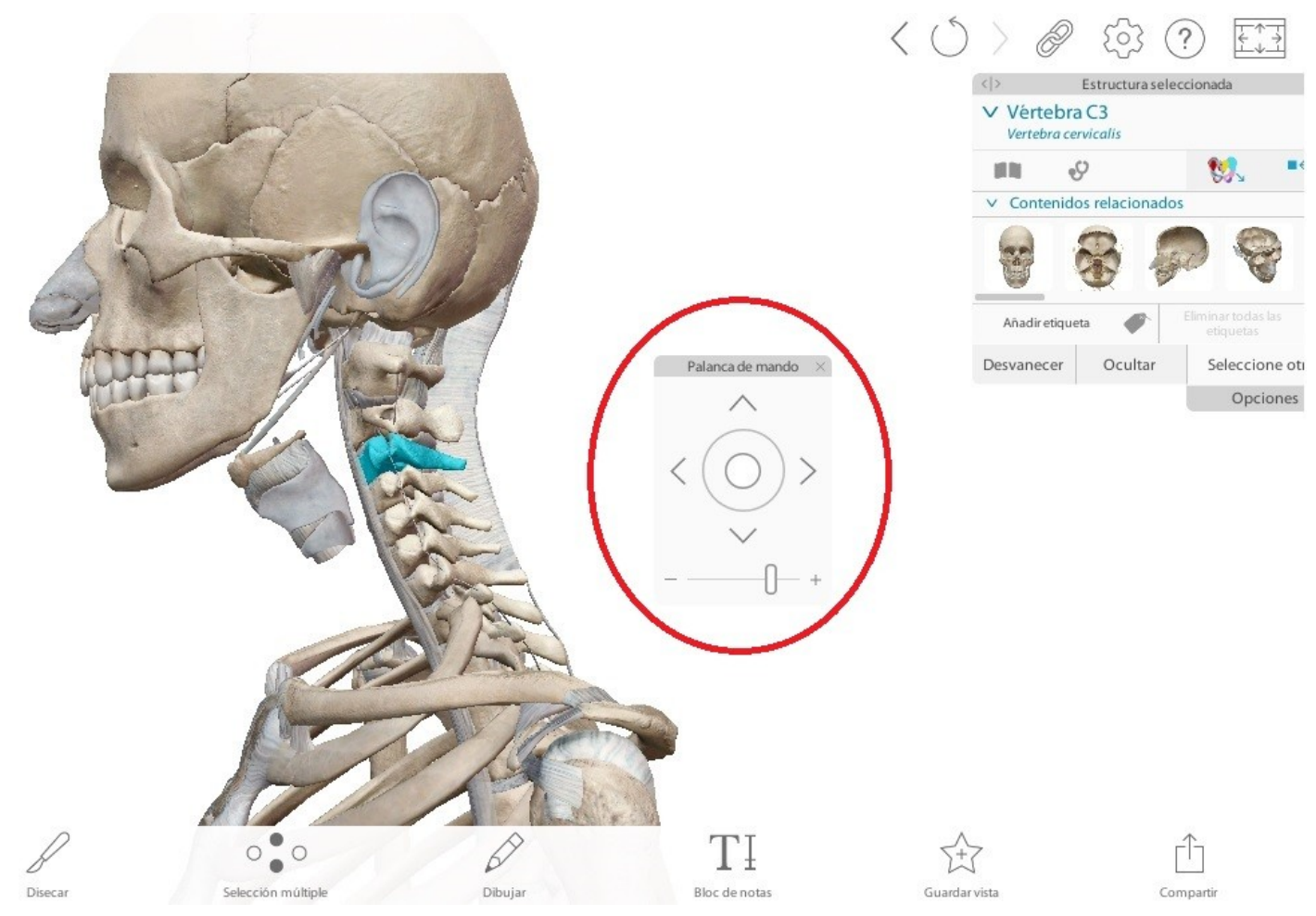

Figura 1. Visible Body. Palanca de mando

Esta palanca concentra las tres operaciones básicas: escalado, desplazamiento y rotación. El escalado (zoom) se realiza con el deslizador en la parte inferior. El desplazamiento de la estructura anatómica completa hacia cuatro direcciones (arriba, abajo, izquierda y derecha) se realiza con los ángulos que apuntan en esas direcciones, y el movimiento de rotación restringido se logra arrastrando el círculo del centro hacia alguna de las cuatro direcciones. Si el circulo es arrastrado hacia arriba o hacia abajo, el objeto rota únicamente sobre el eje $x$, si el círculo es arrastrado hacia izquierda o derecha, el objeto rota únicamente sobre el eje y, mostrando planos canónicos en ambos casos. En cambio, si el círculo es arrastrado hacia posiciones oblicuas intermedias, libremente, o sin prestar atención, se producen rotaciones en planos no canónicos.

\section{Planteamiento y Objetivos.}

Hemos observado durante las clases de introducción al software de anatomía que en tareas simples como capturar una imagen para incrustarla en un documento, los estudiantes se muestran ansiosos por realizar una captura rápida como lo hacen habitualmente con otras App. Sin embargo, teniendo en cuenta las limitaciones de la memoria para retener información, sería recomendable restringir la exposición a imágenes que concuerden con los planos más informativos y esto se logra con rotaciones y vistas canónicas. Pero, aunque los estudiantes estén al tanto de que existen controles de rotación, suelen descartarlos y prefieren la exploración espontánea con el ratón. Por ejemplo, al usar Openanatomy es común que los estudiantes eliminen los planos canónicos internos por considerarlos un elemento extraño a la estructura anatómica que quieren observar. Sin embargo, estos planos cumplen la importante función, especialmente durante las rotaciones, de conservar el marco de referencia de los objetos en movimiento.

Argumentamos que la subestimación de los controles por parte de los estudiantes, está relacionada con una falta de conciencia sobre las propias limitaciones cognitivas en relación a los medios 3D, a saber: la limitación de espacio en la memoria humana para la 
manipulación de imágenes, y la necesidad de nuestro sistema cognitivo de establecer planos canónicos para asegurar la retención. De contar con este conocimiento, al que haremos referencia como metacognición $3 D$, los estudiantes estarían motivados a usar los controles de rotación y las referencias de orientación suministrados por las aplicaciones de software, autorregulándose durante la exploración. Este "alivio" en la carga cognitiva, reduciría el efecto de desorientación (observado tanto en nuestra experiencia áulica como en la literatura científica) y su impacto redundaría en una mayor aceptación del software.

Nuestra hipótesis de trabajo es que hay una influencia de la metacognición $3 D$ en la aceptación del software y proponemos medirla mediante un modelo de aceptación de tecnología.

\section{Métodos}

\subsection{Participantes}

Setenta estudiantes de grado de la facultad de medicina de una universidad de Rosario, Argentina, colaboraron voluntariamente en la investigación y fueron asignados a una de dos condiciones: meta cognición 3D y grupo control. La asignación no fue completamente al azar, ya que los participantes estaban organizados en 4 comisiones para el dictado de las clases, dos comisiones formaron parte del grupo experimental, y otras dos comisiones, del grupo control.

\subsection{Materiales}

Se utilizaron instalaciones informáticas con un proyector y una computadora por alumno, acceso a las aplicaciones Open Anatomy, Anatomy Learning, Anatomie3D, desde Internet, Visible Body desde los repositorios de la universidad, y 3D Slicer que fue descargado previamente en los equipos. Se entregó a cada alumno un texto con descripciones de las aplicaciones de anatomía 3D e indicaciones de trabajo.

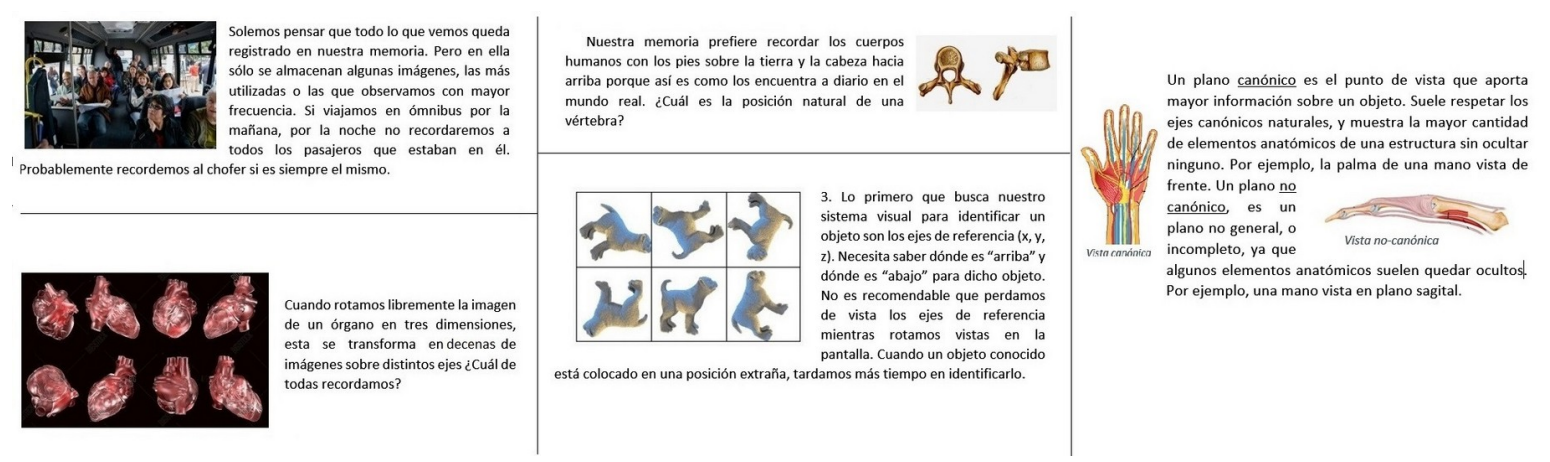

Figura 2. Ejemplos de ítems en el entrenamiento en orientación espacial.

\subsection{Procedimiento.}

Los estudiantes de ambos grupos participaron de un curso de tres encuentros en el gabinete de informática de la universidad, donde se les presentaron las características generales de varias aplicaciones de anatomía 3D, una de ellas suministrada por la institución, y otras de acceso libre en Internet. En el primer encuentro pudieron ver ejemplos de representaciones anatómicas. Durante el segundo encuentro resolvieron una actividad práctica cuya consigna indicaba mirar un vídeo sobre trastornos músculo esqueléticos, elegir una aplicación 3D (pudiendo ser más de una), encontrar en la aplicación las partes anatómicas implicadas en la patología exhibida en el video, capturar tres imágenes en plano frontal, sagital y transversal e incrustarla en un texto. Para lograr 
este propósito, debieron rotar los elementos en pantalla hasta obtener los planos indicados. Sólo los participantes del grupo experimental recibieron, además de las indicaciones básicas para utilizar las aplicaciones, un texto de tres páginas conteniendo ejemplos que ilustraban nociones básicas: capacidad limitada de la memoria, planos canónicos, referencias de orientación espacial (Figura 2). Conforme el docente avanzó en la lectura del texto, fue dando ejemplos de dichas nociones en las aplicaciones 3D, haciendo notar cuáles son los controles de orientación espacial en cada una de ellas. Los participantes en la condición de control fueron entrenados para utilizar las mismas aplicaciones de software pero no recibieron información sobre metacognición (memoria, planos canónicos, etc.). Si bien se les indicó cuales eran los controles disponibles en cada aplicación, no se aclaró cuál es su importancia durante la exploración.

En el último encuentro, los participantes de ambos grupos presentaron su trabajo práctico e hicieron consultas previas a la entrega, con posterioridad a la cual completaron un cuestionario (tabla 1) según el modelo TAM (Technology Acceptance Model) de aceptación de tecnología (19). El cuestionario evalúa la probabilidad de uso del software en base a dos dimensiones: utilidad percibida (UP) y facilidad de uso percibida (FUP). La UP mide el grado en que una persona percibe que la aplicación le será útil para realizar sus tareas, y la FUP mide el esfuerzo o la dificultad que esa persona percibe en la utilización del software. Las preguntas del cuestionario original fueron levemente adaptadas para evaluar las App de anatomía en 3D. El rango de puntuaciones se muestra en la tabla 2. En la tabla 3 se muestran las puntuaciones agrupadas en 3 categorías de aceptación. Se utilizó el programa SPSS para el análisis estadístico, comparando las medias con la t de Student, excepto cuando se indica en el texto. La prueba de Levene se usó para determinar falta de homocedasticidad. Los datos se expresan como media \pm desviación estándar.

Tabla 1. Ítems del cuestionario TAM.

\begin{tabular}{|c|c|}
\hline \multicolumn{2}{|r|}{ Dimensión: Utilidad percibida (UP) } \\
\hline UP1 & $\begin{array}{l}\text { Mi objetivo de aprender anatomía me sería muy difícil de lograr sin las App de } \\
\text { anatomía 3D. }\end{array}$ \\
\hline UP2 & $\begin{array}{l}\text { El uso de las App de anatomía 3D me dan un mayor control sobre mi trabajo de } \\
\text { localización de elementos anatómicos específicos. }\end{array}$ \\
\hline UP3 & $\begin{array}{l}\text { El uso de las App de anatomía 3D me orientan de manera ordenada en mi exploración } \\
\text { del cuerpo humano. }\end{array}$ \\
\hline UP4 & El uso de las App de anatomía 3D mejoran mi desempeño en mis exámenes. \\
\hline UP5 & Las App de anatomía 3D resuelven mis necesidades de estudiante \\
\hline UP6 & Usar las App de anatomía 3D me ahorra tiempo de estudio. \\
\hline UP7 & Con las App de anatomía 3D realizo mis tareas de anatomía más rápidamente. \\
\hline UP8 & Con las App de anatomía 3D puedo realizar tareas que de otro modo serían imposibles. \\
\hline \multicolumn{2}{|r|}{ Dimensión: Facilidad de Uso Percibida (FUP) } \\
\hline FUP1 & No me confundo en mi exploración con las App de anatomía 3D. \\
\hline FUP2 & $\begin{array}{l}\text { No necesito ayudas o explicaciones durante mi exploración del cuerpo humano con } \\
\text { App de anatomía 3D. }\end{array}$ \\
\hline FUP3 & Interactuar con las App de anatomía 3D no requiere esfuerzo de concentración. \\
\hline FUP4 & $\begin{array}{l}\text { Me resulta fácil encontrar en una App de Anatomía el punto anatómico que estoy } \\
\text { buscando. }\end{array}$ \\
\hline FUP5 & $\begin{array}{l}\text { Me resulta sencillo con las App de anatomía ser consciente de qué parte anatómica } \\
\text { estoy observando. }\end{array}$ \\
\hline FUP6 & $\begin{array}{l}\text { Me resulta sencillo con las App de anatomía capturar información en su orientación } \\
\text { correcta. }\end{array}$ \\
\hline
\end{tabular}


Tabla 2. Opciones de respuesta y puntuación

\begin{tabular}{|l|c|}
\hline \multicolumn{1}{|c|}{ Opciones } & Puntos \\
\hline Muy de acuerdo & 5 \\
\hline De acuerdo & 4 \\
\hline Neutral & 3 \\
\hline En desacuerdo & 2 \\
\hline Muy en desacuerdo & 1 \\
\hline
\end{tabular}

\section{Resultados}

Las puntuaciones generales de aceptación (UP) de las aplicaciones de anatomía 3D (Figura 3), considerando el grupo completo (70 alumnos), es favorable en un $85,7 \%$, muy favorable en un $10 \%$ y desfavorable en un $4,3 \%$, teniendo en cuenta el criterio de agrupamiento según la tabla 3.

Tabla 3. Criterio de agrupamiento.

\begin{tabular}{|c|c|}
\hline Puntos & $\begin{array}{c}\text { Etiqueta de } \\
\text { agrupamiento }\end{array}$ \\
\hline $1-2$ & Muy Desfavorable \\
\hline $2-3$ & Desfavorable \\
\hline $3-4$ & Favorable \\
\hline $4-5$ & Muy Favorable \\
\hline
\end{tabular}

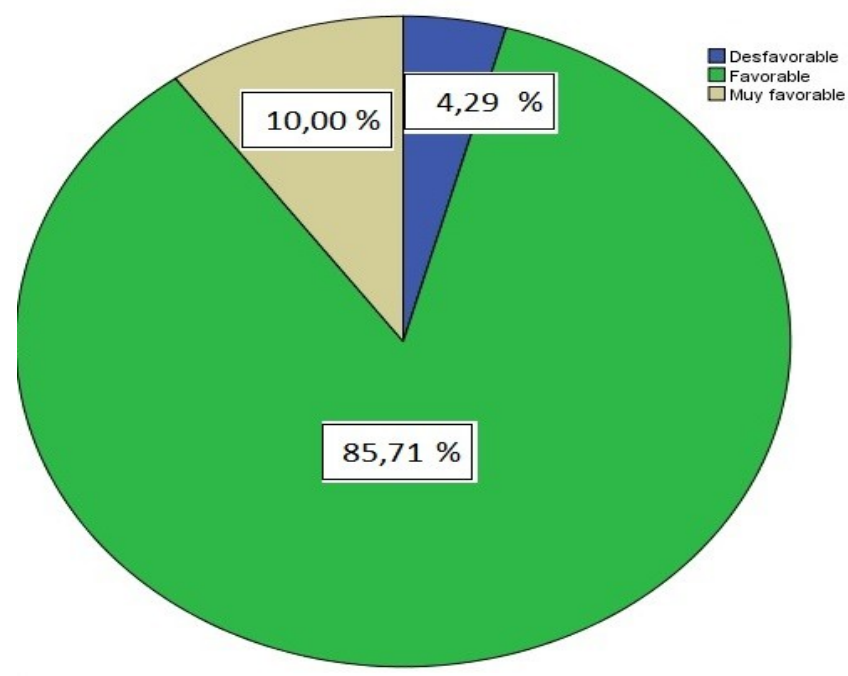

Figura 3. Utilidad percibida (agrupado)

Se analizaron las diferencias entre grupos calculando la UP como la media agrupada de las puntuaciones, observando una diferencia significativa en la utilidad percibida. La media de UP fue mayor en el grupo entrenado en metacognición $(4.75 \pm 0.29)$ que en el grupo sin entrenamiento $(4.52 \pm 0.31)$. Sin embargo, no se observa diferencia significativa en la facilidad de uso percibida. La media de FUP fue mayor en el grupo entrenado (4.41 \pm $0.35)$ que en el grupo control $(4.25 \pm 0.30)$. 
Contrastando ítems específicos, puede observarse una concentración de las diferencias entre grupos en las variables UP2, UP3 y FUP6 a favor del grupo experimental. En el caso de UP2, "El uso de las App de anatomía 3D me dan un mayor control sobre mi trabajo de localización de elementos anatómicos específicos", la media fue superior en el grupo experimental $(4.03 \pm 0.17)$ que en el grupo control $(3.35 \pm 0.77)$. La UP3, "El uso de las App de anatomía 3D me orientan de manera ordenada en mi exploración del cuerpo humano", sin homocedasticidad de varianza, igualmente con una media mayor en el grupo entrenado $(4.05 \pm 0.53)$ que en el grupo control $(3.05 \pm 0.88)$, y la FUP6, "Me resulta sencillo con las App de anatomía capturar información en su orientación correcta.", también mayor en el grupo entrenado $(3.64 \pm 0.77)$ que en el grupo control $(2 \pm 0.82)$.

La diferencia anterior se compensa en parte con el caso de FUP2 y FUP3 que resultan aparentemente contradictorios. Los estudiantes del grupo experimental fueron guiados por el docente para descubrir que sus propias exploraciones pueden ser reguladas mediante un esfuerzo mayor de concentración y mediante el uso de controles que "lentifican" la exploración produciendo un beneficio. En este caso, una mayor "lentitud" no es sinónimo de ineficiencia, sino de reflexión y precaución. Esto podría explicar el descenso del acuerdo con el ítem FUP2, "No necesito ayudas o explicaciones durante mi exploración del cuerpo humano con App de anatomía 3D" porque los estudiantes revalorizan las explicaciones. La diferencia en la variable FUP2 es a favor del grupo control, que trabajó libremente y más rápido, por ignorar los problemas de la rotación libre y prescindir de las explicaciones del docente. La media de FUP3 fue mayor en el grupo control $(3.74 \pm 0.67)$ que en el grupo entrenado $(2,69 \pm 0.98)$. Así, FUP3, "Interactuar con las App de anatomía 3D no requiere esfuerzo de concentración", es otra diferencia a favor del grupo control que ha realizado rotaciones libres sin demasiada concentración y sin advertir diferencias. La media de FUP3 fue mayor en el grupo control $(3.74 \pm 0.51)$ que en el grupo entrenado $(2,81 \pm 0.98)$.

\section{Discusión}

Esta investigación comparó el nivel de aceptación del software de anatomía 3D de alumnos con preparación metacognitiva para el uso de las aplicaciones vs. alumnos autodidactas. Los primeros, recibieron información acerca de las limitaciones de su propia memoria en interacción con imágenes 3D y de estrategias para la rotación regulada de los planos. Los participantes del grupo control, en cambio, se condujeron de manera espontánea, efectuando las rotaciones con movimientos libres del ratón como lo harían con cualquier otra App.

Todos los alumnos debieron rotar imágenes hasta obtener las vistas canónicas especificadas en la consigna. Los alumnos que utilizaron controles de rotación tuvieron más ventajas para obtener las vistas canónicas pero eso no implicó una mayor velocidad en la resolución del trabajo, todo lo contrario. Realizar rotaciones más planificadas y obtener mejores vistas implica una mayor inversión de tiempo. No sorprende que hayan manifestado una mayor aceptación desde el punto de vista de la utilidad percibida (UP) pero puntuando más bajo en la facilidad de uso (FUP). Las medidas de usabilidad del software se basan en la agilidad: el objetivo es que los usuarios no incurran en demoras ni necesiten ayuda externa.

Por el contrario, el objetivo pedagógico de desarrollar la capacidad metacognitiva para operar con representaciones 3D exige una mayor demanda de tiempo para la autorregulación en la exploración y el uso de controles de orientación. Esto conduce a dos conclusiones. 
Por un lado, el modelo TAM podría no ser la herramienta más apropiada para medir la aceptación de software con conceptos tan complejos como la anatomía humana, lo que constituye una de las debilidades de este estudio, junto con el bajo número de participantes.

Las velocidades de las interfaces digitales no son compatibles con las del desarrollo de habilidades cognitivas que se necesitan para operar con dichas interfaces. Esto podría sugerir que ciertas capacidades se deben desarrollar antes de operar con estos medios. El caso de anatomía 3D podría ser uno de los muchos casos de aprendizaje con tecnologías que demandan conocimiento metacognitivo.

A pesar de los esfuerzos de los gobiernos para que las TIC sean accesibles a todos los alumnos, algunos estudios vienen mostrando que no es clara la correlación entre el uso de herramientas digitales y el desempeño (20-22). Al parecer, las habilidades para operar con las TIC se podrían adquirir más fácilmente si los estudiantes ya tuvieran competencia en procesos superiores de pensamiento. Un requisito que podría asegurarse si se incorporaran estrategias de enseñanza con atención a procesos metacognitivos, en otras palabras, si el profesor de informática pudiera enseñar a pensar antes de usar.

\section{Conclusiones.}

- El estudio realizado pone en evidencia la necesidad de una mayor participación del docente en el aprendizaje de la manipulación de tecnologías 3D de anatomía

- Es importante también tener un conocimiento más profundo de los procesos cognitivos que intervienen en dicha actividad, no sólo por parte del docente sino del alumno, para autoregular su propio aprendizaje.

- Las habilidades para operar con TIC se podrían adquirir más fácilmente si los estudiantes ya tuvieran competencia en procesos superiores de pensamiento. Un requisito que podría asegurarse si se incorporaran estrategias de enseñanza con atención a procesos metacognitivos, en otras palabras, si el profesor de informática pudiera enseñar a pensar antes de usar.

Financiación: No ha habido financiación

Declaración de conflicto of interés: Los autores declaran no tener ningún conflicto de intereses.

\section{Referencias}

1 Prensky M. Digital Natives, Digital Immigrants Part 1. Horiz. septiembre de 2001;9(5):1-6. https:/ / www.marcprensky.com/writing/Prensky\%20-\%20Digital\%20Natives,\%20Digital\%20Immigrants \%20-\%20Part1.pdf

2 Duarte A. Aproximación al estudio de la cognición. Publicación del Centro de Estudiantes de Psicología Universidad de Buenos Aires. 1986.

3 Burin D. Aníbal Duarte. Subj. procesos cogn. 2010; 14(1). Disponible en: http:/ / www.scielo.org.ar/scielo.php?script=sci arttext\&pid=S1852-73102010000100002.

4 Preim B, Saalfeld P. A survey of virtual human anatomy education systems. Comput Graph. 1 de abril de 2018;71:132-53. https:// doi.org/10.1016/j.cag.2018.01.005.

5 Bergman EM, Prince KJ, Drukker J, van der Vleuten CP, Scherpbier AJ. How much anatomy is enough? Anat Sci Educ. 2008 Jul-Aug;1(4):184-8. http://doi.org/10.1002/ase.35

6 McKeown PP, Heylings DJ, Stevenson M, McKelvey KJ, Nixon JR, McCluskey DR. The impact of curricular change on medical students' knowledge of anatomy. Med Educ. 2003 Nov;37(11):954-61. http://doi.org/10.1046/j.1365-2923.2003.01670.x

7 Spielmann PM, Oliver CW. The carpal bones: a basic test of medical students' and junior doctors' knowledge of anatomy. Surgeon. 2005 Aug;3(4):257-9. http://doi.org/10.1016/s1479-666x(05)80087-3 
8 Waterston SW, Stewart IJ. Survey of clinicians' attitudes to the anatomical teaching and knowledge of medical students. Clin Anat. 2005 Jul;18(5):380-4. http://doi.org/10.1002/ca.20101

9 Garg A, Norman GR, Spero L, Maheshwari P. Do virtual computer models hinder anatomy learning? Acad Med. 1999 Oct;74(10 Suppl):S87-9. http://doi.org/10.1097/00001888-199910000-00049

10 Garg AX, Norman GR, Eva KW, Spero L, Sharan S. Is there any real virtue of virtual reality?: the minor role of multiple orientations in learning anatomy from computers. Acad Med. 2002 Oct;77(10 Suppl):S97-9. http://doi.org/10.1097/00001888-200210001-00030

11 Huk T. Who benefits from learning with 3D models? the case of spatial ability. J Comput Assist Learn. 2006;22(6):392-404. https://doi.org/10.1111/j.1365-2729.2006.00180.x

12 Khot Z, Quinlan K, Norman GR, Wainman B. The relative effectiveness of computer-based and traditional resources for education in anatomy. Anat Sci Educ. 2013 Jul-Aug;6(4):211-5. http://doi.org/10.1002/ase.1355

13 Levinson AJ, Weaver B, Garside S, McGinn H, Norman GR. Virtual reality and brain anatomy: a randomised trial of e-learning instructional designs. Med Educ. 2007 May;41(5):495-501. http://doi.org/10.1111/j.1365-2929.2006.02694.x

14 Berney S, Bétrancourt M. Learning Three-Dimensional Anatomical Structures with Animation: Effect of Orientation References and Learners' Spatial Ability. En: Learning from Dynamic Visualization: Innovations in Research and Application. 2017.

15 Mayer R. Guiding Cognitive Processing During Learning with Animations: Commentary on Parts III and IV. En: Learning from Dynamic Visualization: Innovations in Research and Application. 2017.

16 Stull A, Hegarty M, Mayer R. Anatomy Learning with Virtual Objects. En: AAAI Spring Symposium Technical Report. 2010.

17 Stull AT, Hegarty M, Mayer RE. Getting a handle on learning anatomy with interactive three-dimensional graphics. J Educ Psychol. 2009;101(4):803-16.https:/ / doi.org/10.1037/a0016849

18 Hegarty M. Chapter 7 - Components of Spatial Intelligence. En: Psychology of Learning and Motivation [Internet]. Academic Press; 2010 [citado 5 de marzo de 2021]. p. 265-97. (The Psychology of Learning and Motivation; vol. $52)$. Disponible en: https://www.sciencedirect.com/science/article/pii/S0079742110520073

19 Davis FD. Perceived Usefulness, Perceived Ease of Use, and User Acceptance of Information Technology. MIS Quarterly. 1989;13(3):319-40. https:/ / doi.org/10.2307/249008

20 OECD (2015), Students, Computers and Learning: Making the Connection, PISA, OECD Publishing. https://www.oecd.org/publications/students-computers-and-learning-9789264239555-en.htm

$21 \mathrm{Hu}, \mathrm{C}$. Students, computers and learning: Where is the connection? Educ Inf Technol 22, 2665-2670 (2017). https://www.oecd.org/publications/students-computers-and-learning-9789264239555-en.htm

22 Schmid, Regina; Petko, Dominik (2019). Does the use of educational technology in personalized learning environments correlate with self-reported digital skills and beliefs of secondary-school students? Computers and Education, 136:75-86. https://doi.org/10.1016/j.compedu.2019.03.006 


\section{Anexo}

\section{Origen de las App de anatomía.}

El origen de estas aplicaciones se remonta a los inicios del proyecto Visible Human en 1986 (1): más de un millón de dólares invertidos en la captación de imágenes radiológicas del cuerpo humano habilitaron la creación de representaciones tridimensionales completas, anatómicamente detalladas y de libre acceso. Se proporcionó una biblioteca de dominio público de imágenes de criosección transversales, tomografía computarizada y resonancia magnética, a partir de las cuales, numerosos usuarios desarrollaron aplicaciones para explotar dichas bases de datos, por ejemplo, con fines educativos. Los libros de anatomía (atlas), piedra angular de la educación médica durante cientos de años, primeros libros de referencia constante para los estudiantes de medicina y para los médicos de todo el mundo, han ido mejorando paulatinamente la calidad de las representaciones gráficas pero se han caracterizado por su costo elevado. En cambio, los atlas digitales de anatomía disponibles en la web, o en los teléfonos celulares, han complementado (aunque no reemplazado) los libros impresos brindando información anatómica a cualquier persona, incluso al público en general, de una manera más ágil.

\section{Ventajas de las App de anatomía.}

La posibilidad de acceso a la manipulación visual de información anatómica asociada al contenido conceptual, representa una oportunidad valiosa de aprendizaje relajado, sin pérdida de exhaustividad, que podría acercar a los alumnos a los conceptos anatómicos desde una base segura para ensayar sin riesgo. Los estudiantes pueden simular y practicar la resolución de problemas relacionados con alguna patología, ilustrándola, explicándola y gestionando la documentación bibliográfica para argumentar y justificar sus decisiones. Los atlas digitales permiten mover espacialmente un objeto, incluso a posiciones de difícil acceso en el mundo real, activar, resaltar u ocultar sistemas articulares, musculares o algunas de sus partes, clasificarlos, incluso producir un modelo anatómico real mediante impresión en 3D.

\section{Procesamiento cognitivo de la información visual}

\subsection{Distinción entre imágenes externas e imágenes internas}

Hegarty (2) definió y comparó los conceptos de visualización externa y visualización interna: una visualización externa es una imagen en un papel o en la pantalla de una computadora que puede ser observada por un individuo, mientras que una visualización interna es una representación en la mente de un individuo. Algunas profesiones, como la medicina, dependen en gran medida de la capacidad de representación de visualizaciones internas. Por ejemplo, un estudiante de kinesiología durante la exposición de un caso clínico, podría describir lo siguiente:

"La paciente presenta un dolor agudo, localizado, punzante, en la parte lateral, posterior e interna del brazo derecho, que se agrava al levantar el brazo en abducción por encima de los $90^{\circ}$, al levantar peso o al realizar rotación externa. Asociamos los síntomas con la cirugía reciente del tendón supraespinoso".

Al egresar de la universidad, en su práctica clínica, los profesionales dependerán de un conocimiento relacional pormenorizado de la anatomía humana que les debe permitir evocar nítidamente sus propias representaciones mentales espaciales de dichas estructuras, esto es, la forma anatómica (en el ejemplo, el brazo), la posición (e.g. lateral, 
posterior, interna), la dirección del movimiento (e.g. abducción por encima de los $90^{\circ}$, rotación externa), o cualquier otro proceso, como encontrar una vena para inyectar un analgésico o comprender una imagen médica. La interpretación de imágenes radiológicas se basa en representaciones espaciales internas a partir de las cuales se infieren las partes anatómicas de un cuerpo humano real. Los cirujanos poseen modelos mentales internos que les permiten planificar una estrategia para moverse a través de la zona operada.

\subsection{Planos canónicos}

Cuando vemos un objeto conocido en su posición habitual lo identificamos tan rápidamente que no nos percatamos de ningún esfuerzo (Fig. 1, izquierda), pero es posible que notemos cierta demora al tratar de identificar el mismo objeto en una posición extraña o distinta a la habitual (Fig. 1, derecha).
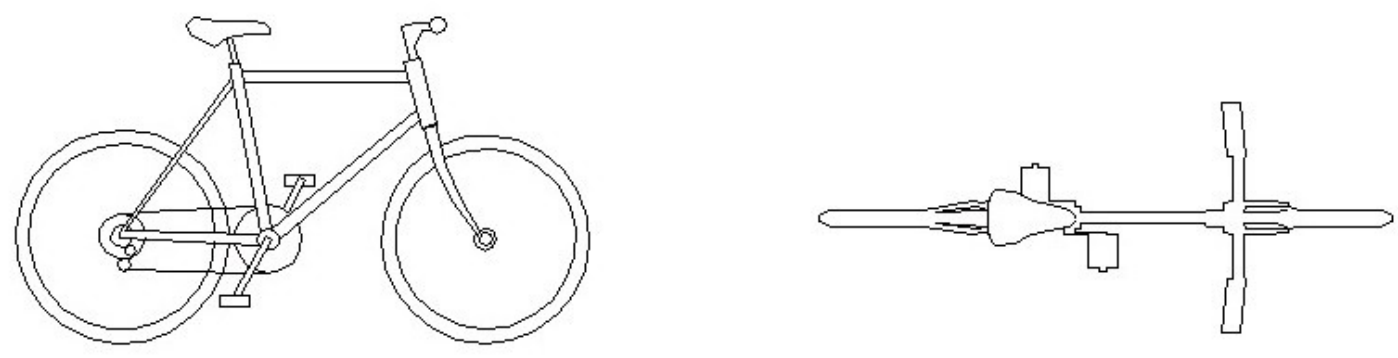

Figura 1. Bicicleta desde plano canónico (izquierda) y plano no canónico.

Los objetos del mundo real se presentan en tres dimensiones, por lo tanto, pueden ser observados desde cualquier ángulo. Las primeras teorías sobre la representación de objetos tridimensionales en memoria postularon que se almacena una representación en tres dimensiones con independencia del punto de vista (3). De ser así, al observar un objeto conocido, desde cualquier ángulo, el observador haría coincidir la imagen externa con su imagen interna instantáneamente, sin embargo, se ha constatado que el tiempo de reconocimiento depende sistemáticamente de la orientación del estímulo (4-8). Es decir, la observación de objetos en posiciones extrañas (no canónicas) implica mayor tiempo de reconocimiento. Durante ese tiempo se realiza una acomodación (transformación) del sistema de coordenadas percibido para adaptarlo al marco de referencia conocido, es decir, para alinear la imagen externa con la imagen interna (en MLP) (9). En dicha memoria sólo se almacenan algunas vistas en 2D para cada objeto (10-12). Durante el reconocimiento, el observador busca rasgos salientes que permitan identificar el objeto, al tiempo que alinea ambas imágenes comparando la imagen externa (Fig. 2, izquierda) con la vista 2D más cercana entre las vistas almacenadas en su memoria (Fig. 2, derecha).
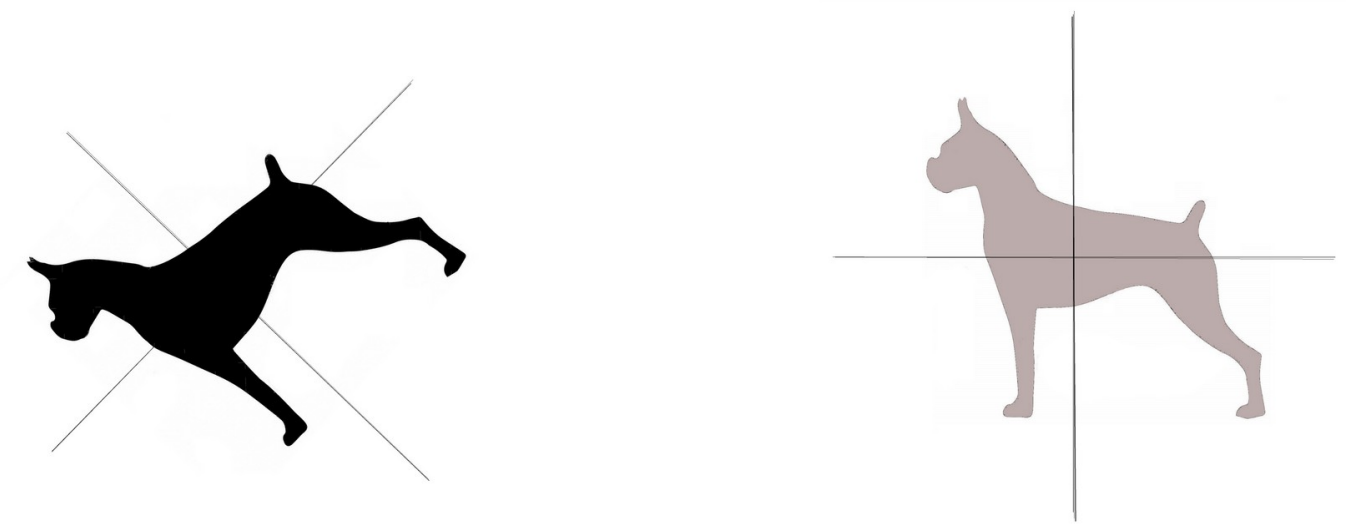

Figura 2. Imagen de perro observada en plano no-canónico e imagen en memoria 
El punto de vista del observador en el reconocimiento de escenas y objetos, influye en su recuerdo posterior de los mismos. Esto es valido para objetos estáticos (10, 13-14), o dinámicos (15). Según Palmer (16), si "diferentes puntos de vista muestran información diferente acerca del objeto", algunos puntos de vista deben mostrar información que tiene mayor coincidencia que otros con las representaciones almacenadas del objeto. Estos puntos de vista que contienen mayor información fueron denominados puntos de vista canónicos, pero no deben confundirse con una posición determinada de observación sino con una "perspectiva privilegiada" o más informativa que depende de cada objeto o escena. Exponerse a todos los puntos de vista de un objeto no garantiza un mejor reconocimiento del objeto real en el próximo encuentro. Observar los planos canónicos de un objeto (los más completos e informativos) es más útil para su memorización que exponerse a rotaciones completas y exhaustivas de todos los puntos de vista, incluso los poco informativos. Esto tiene especial interés para el estudio de anatomía con aplicaciones 3D con posibilidad de rotación ilimitada. Los atlas de anatomía tradicionales en libros de texto muestran elementos anatómicos únicamente desde ciertos ángulos clave, por ejemplo, los huesos carpianos y sus ligamentos podrían presentarse desde una vista anterior (Fig. 3, izquierda) y una posterior (Fig. 3, centro). En contraste, una vista inferior desde las falanges distales sería poco informativa sobre la estructura general (Fig. 3, derecha).
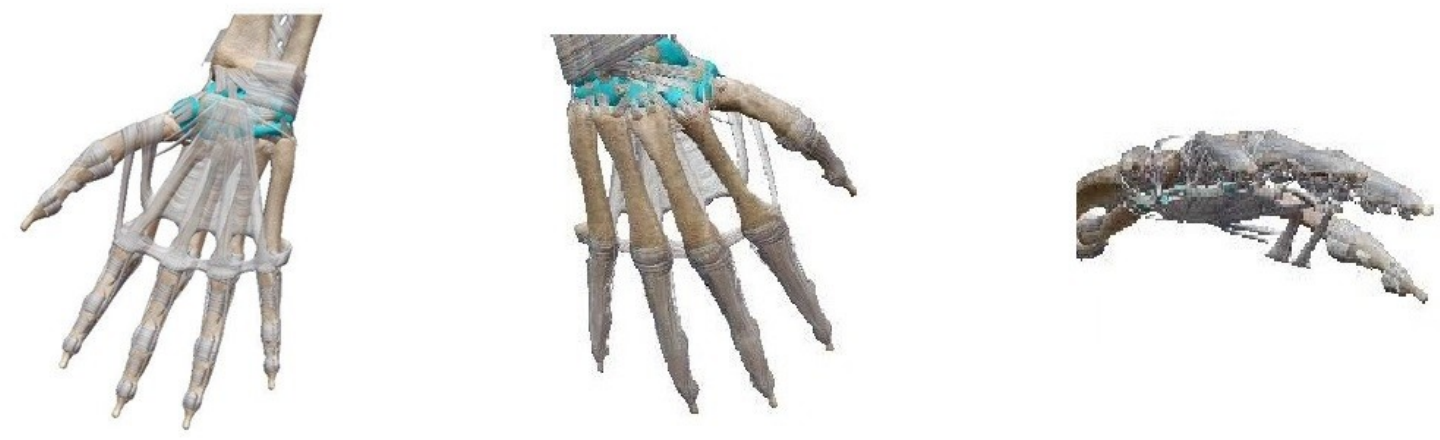

Figura 3. Falanges y ligamentos desde dos planos canónicos y un plano no canónico.

\section{Dificultades vinculadas a la inteligencia espacial}

Está establecido que la inteligencia espacial difiere de un individuo a otro. Hegarty (17) distingue dos factores en la inteligencia espacial: capacidad de visualización y capacidad meta-representacional. El primero refiere a la capacidad de construir imágenes mentales, manipularlas, simular procesos mentalmente y comprender las relaciones espaciales entre los elementos percibidos. Esta capacidad se ha medido mediante diversas pruebas, como la rotación mental, o el plegado de papel pero también incluye estrategias de tipo analítico como la descomposición de tareas. Un segundo factor de la inteligencia espacial es la habilidad meta-representacional, es decir, la capacidad de elegir la representación (imagen) externa óptima para una tarea, y el uso efectivo de representaciones externas novedosas, como las 3D interactivas.

A pesar de los beneficios del aprendizaje en 3D, se han documentado desventajas importantes para estudiantes con poca habilidad visoespacial. Para estas personas, visualizar un objeto 3D desconocido desde múltiples puntos de vista sería un desafío que podría incluso impedir el aprendizaje. La dificultad para aprender con estas múltiples vistas no sólo reside en una dificultad para comprender las imágenes (primer factor mencionado) sino para comprender cómo autoregular el uso de imágenes digitales eligiendo los puntos de vista más informativos. Aunque los estudiantes tengan la libertad para explorar y dispongan de controles de exploración, no los usan eficientemente para 
observar los aspectos estructurales de las imágenes. Keehner y colaboradores (18), ofrecieron una aplicación con mayor control interactivo a los estudiantes durante la exploración por computadora y demostraron que la autonomía y la interactividad per se no son garantía de mejor desempeño en el razonamiento espacial. Existen diferencias individuales en la eficacia con la que las personas utilizan los controles de las visualizaciones (por ejemplo, rotar, escalar) y en la medida en que aprovechan las ayudas externas proporcionadas por el software. Por lo tanto, diferentes personas obtienen diferente información visual a partir de las mismas imágenes externas, en relación a su habilidad espacial y sus conocimientos previos $(17,19)$. Algunos de ellos obtienen la información clave que es pertinente para el objetivo de la tarea y a otros se les dificulta obtener esa información.

Analizando protocolos en voz alta se pudo constatar la dificultad de los estudiantes con menor habilidad espacial para realizar las mismas tareas:

"El siguiente protocolo de un participante de baja habilidad espacial (...) ilustra la falta de conciencia metacognitiva. Este participante no puede descubrir cómo el uso de la visualización externa puede ayudar con la tarea de la sección transversal. Ella gira el enunciado del problema, impreso en papel (...) para tratar de imaginar una vista diferente del objeto, en lugar de girar la visualización 3D externa que realmente le daría esta vista. Ella comenta que una vez que comienza a rotar la visualización externa se desorienta." (17)

Levinson y colaboradores (20), en un estudio donde los estudiantes aprendían la anatomía superficial del cerebro, descubrieron que las vistas principales del cerebro, presentadas de forma sencilla, eran la manera más eficaz de utilizar el e-learning para enseñar anatomía cerebral.

\section{Referencias de orientación espacial}

Las dificultades observadas en la manipulación del 3D por parte de estudiantes dieron lugar a la incorporación de referencias a la orientación espacial en diversas aplicaciones de software para guiar a los estudiantes hacia los planos canónicos.

Un estudio, Stull y colaboradores (21), tras advertir la desorientación que mostraban algunos alumnos al tener que rotar imágenes virtuales (que resultaba incluso más difícil que rotar objetos reales), demostraron que introducir referencias de orientación visibles en el objeto a rotar mejoraba el aprendizaje. Los participantes debían aprender la estructura de una vértebra mediante la manipulación de un modelo virtual. Giraron el objeto virtual utilizando unas "manijas" que restringían la rotación a un eje, pudiendo realizar rotaciones en tres ejes (tres manijas). Este recurso alivió el desafío de las rotaciones no controladas alrededor de ejes no canónicos. Los autores concluyeron que proporcionar referencias de orientación apoya el aprendizaje y promueve representaciones mentales coherentes de estructuras anatómicas.

\subsection{Control del ratón en la rotación}

Es importante notar que el software que se conoce comúnmente como 3D, no implica una verdadera exploración en tres dimensiones. Se trata de proyecciones sobre una pantalla en dos dimensiones (2D). Si bien existen los ratones o punteros 3D, son más utilizados los tradicionales (2D) que se desplazan sobre una superficie bidimensional para proyectarse en una imagen 3D simulada. Esta limitación tiene como consecuencia que cada desplazamiento sobre el eje $x$ (2D) se interpreta como una rotación del objeto alrededor del eje y 3D, y cada desplazamiento del ratón sobre el eje y (2D) se interpreta 
como una rotación del objeto alrededor del eje x 3D. En general, las personas tienden a mover el ratón en forma circular, los desplazamientos son en ambos ejes ( $\mathrm{x}$ e $\mathrm{y}$ ) simultáneamente, produciendo rotaciones 3D en planos oblicuos, lo que dificulta poder posicionar una imagen en una vista canónica. Para solucionar este problema, se han incorporado diversos controles. En algunas aplicaciones, la velocidad de respuesta del ratón está disminuida (menor cantidad de líneas en pantalla por desplazamiento). A velocidad normal, la rotación es demasiado sensible al ratón por lo que es conveniente modificarla en el sistema operativo.

\subsection{Ejemplos de referencias de orientación en aplicaciones actuales}

\subsubsection{Open anatomy}

El proyecto Open Anatomy (openanatomy.org) es un desarrollo de datos abiertos, colaborativo y de distribución gratuita, todavía en su etapa de desarrollo inicial (22), que está orientado fundamentalmente a la educación y la investigación y ofrece una serie de atlas del cuerpo humano creados con la aplicación 3D Slicer (slicer.org), una plataforma de análisis de imágenes radiológicas para la segmentación y el renderizado volumétrico. Para una rigurosa localización y segmentación, cuenta con referencias de orientación espacial múltiples y muy visibles, que son heredadas por Openanatomy.

Tanto en Openanatomy como en Slicer, las referencias espaciales más robustas son "internas" a la estructura anatómica visualizada. Se trata de tres planos canónicos incrustados en el volumen 3D. Cada uno de estos planos (axial, coronal y sagital) está vinculado con un visor en $2 \mathrm{D}$ que se muestra a un lado de la imagen 3D (Fig. 4, a la derecha). Cada visor contiene el conjunto de cortes en la misma dirección que pueden ser explorados moviendo un control deslizable. A medida que se pasa de un corte a otro, el movimiento se refleja en el avance del plano en la imagen 3D.

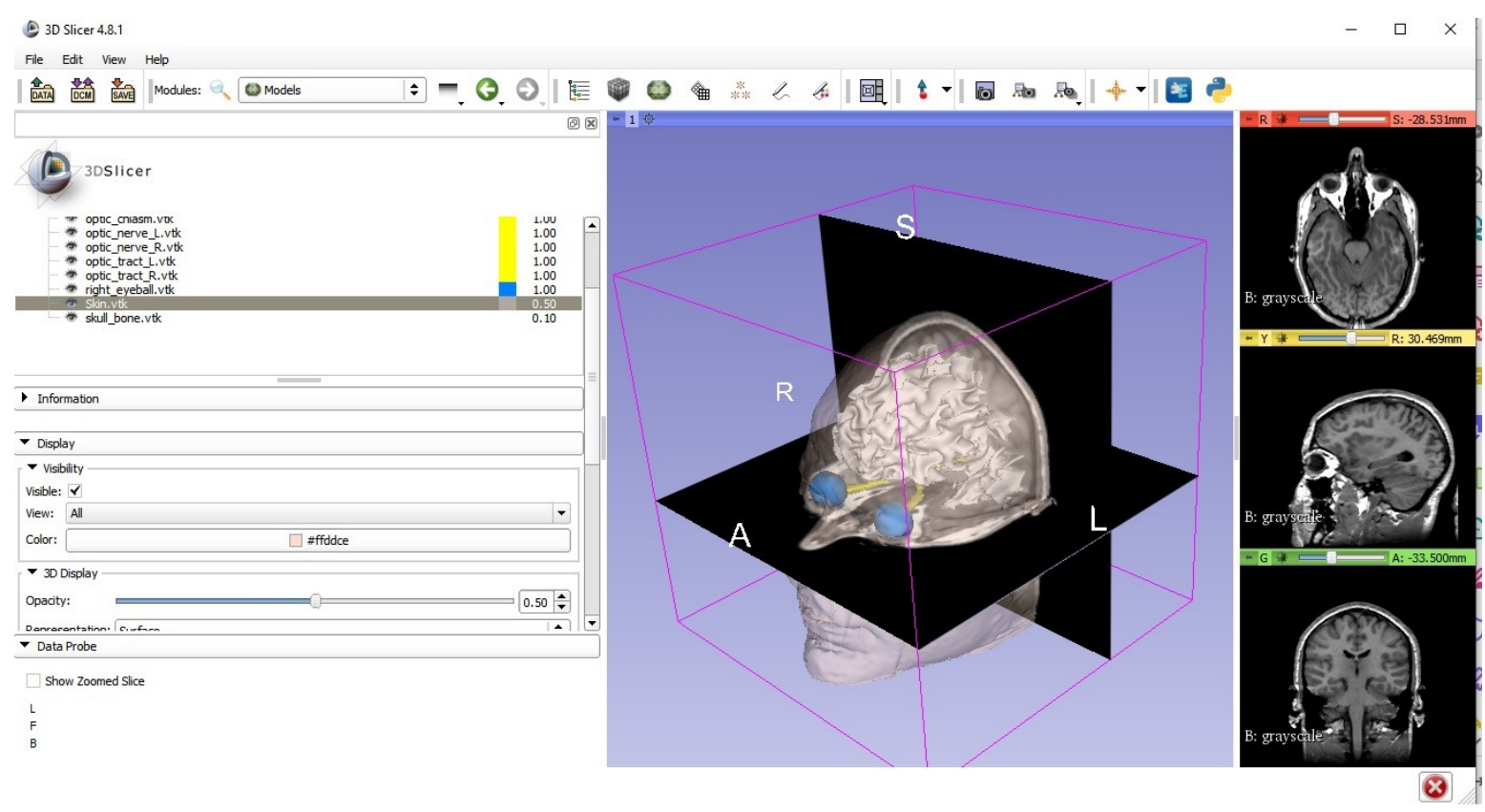

Figura 4. 3D Slicer. Planos y ejes canónicos

Este deslizamiento de los planos permite ubicar una sección específica en el volumen 3D. Con otros controles se pueden invisibilizar las capas para lograr hallar un punto especìfico y observar en detalle. Las vistas anterior $(A)$, posterior $(\mathrm{P})$, superior $(\mathrm{S})$, inferior (I), izquierda (L) y derecha (R) constituyen otra referencia de orientación espacial. En Openanatomy, de finalidad específicamente educativa, se dispone de los mismos planos 
canónicos pero se agrega un controlador de vistas (Fig. 5, abajo, a la izquierda) para rotar el objeto con referencia a las vistas posibles. A pesar de la presencia de estas referencias espaciales es bastante difícil lograr una rotación alrededor de un único eje por medio del ratón tradicional (como se explicó anteriormente) por lo que es recomendable ajustar la velocidad de avance del ratón en el sistema operativo, para lograr movimientos más lentos. En última instancia, si la posición del elemento rotado no es clara, puede utilizarse el ajuste de la vista (Fig. 5, arriba izquierda) para volver a colocar la vista anterior al frente.

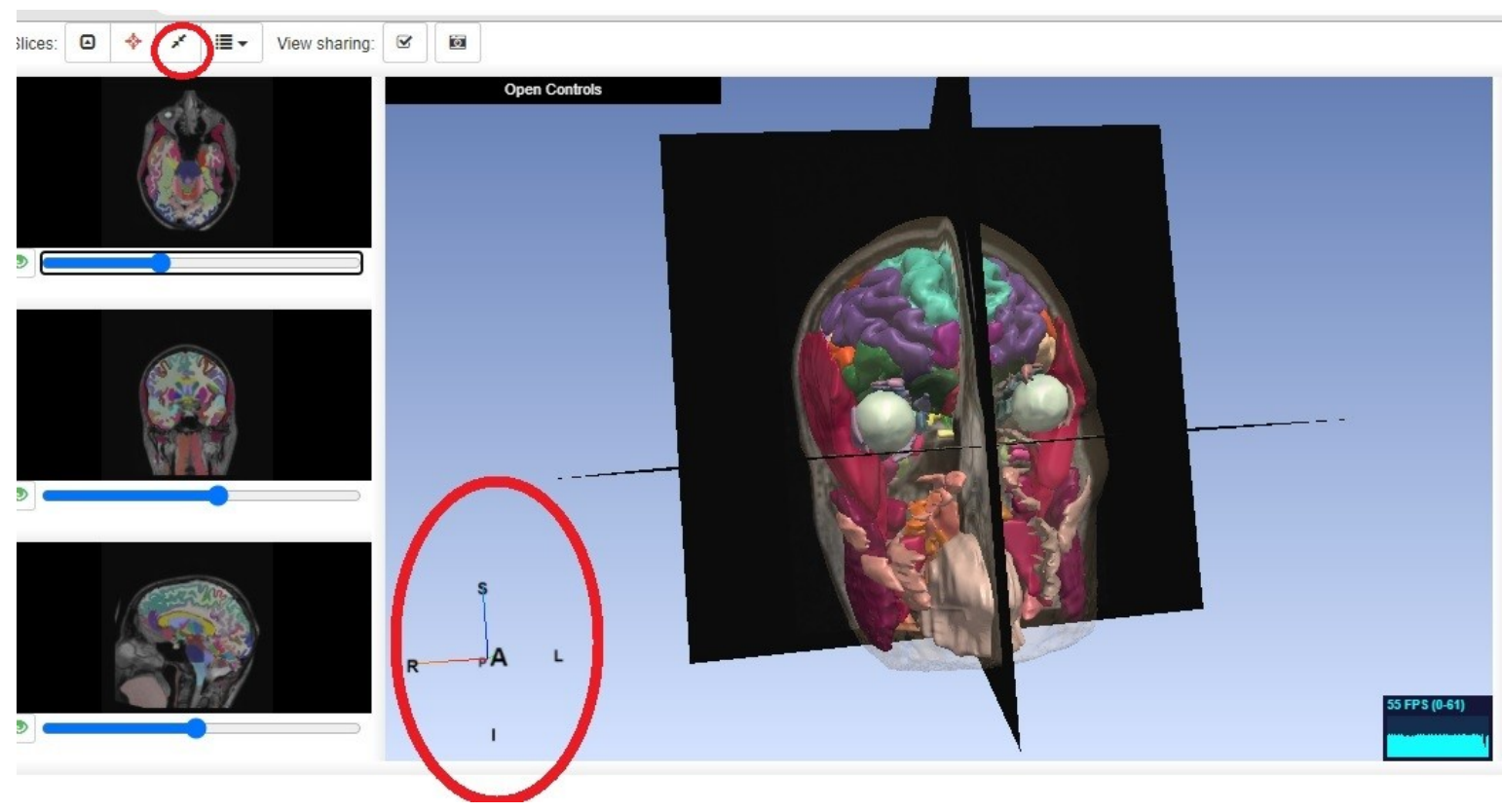

Figura 5. Open Anatomy. Ajuste de imagen y controlador de vistas

Anatomy learning (Fig. 6) es una de las aplicaciones más populares por su atractivo diseño visual y practicidad que contrastan con sus limitados controles de orientación. Contiene un control para forzar un traslado de cualquier vista hacia el frente de la pantalla, por ejemplo, pulsando en A (cubo), la vista anterior se posiciona al frente, pulsando en $\mathrm{P}$, la vista posterior se posiciona al frente. Este control aparece como menú contextual dando clic derecho sobre el fondo.

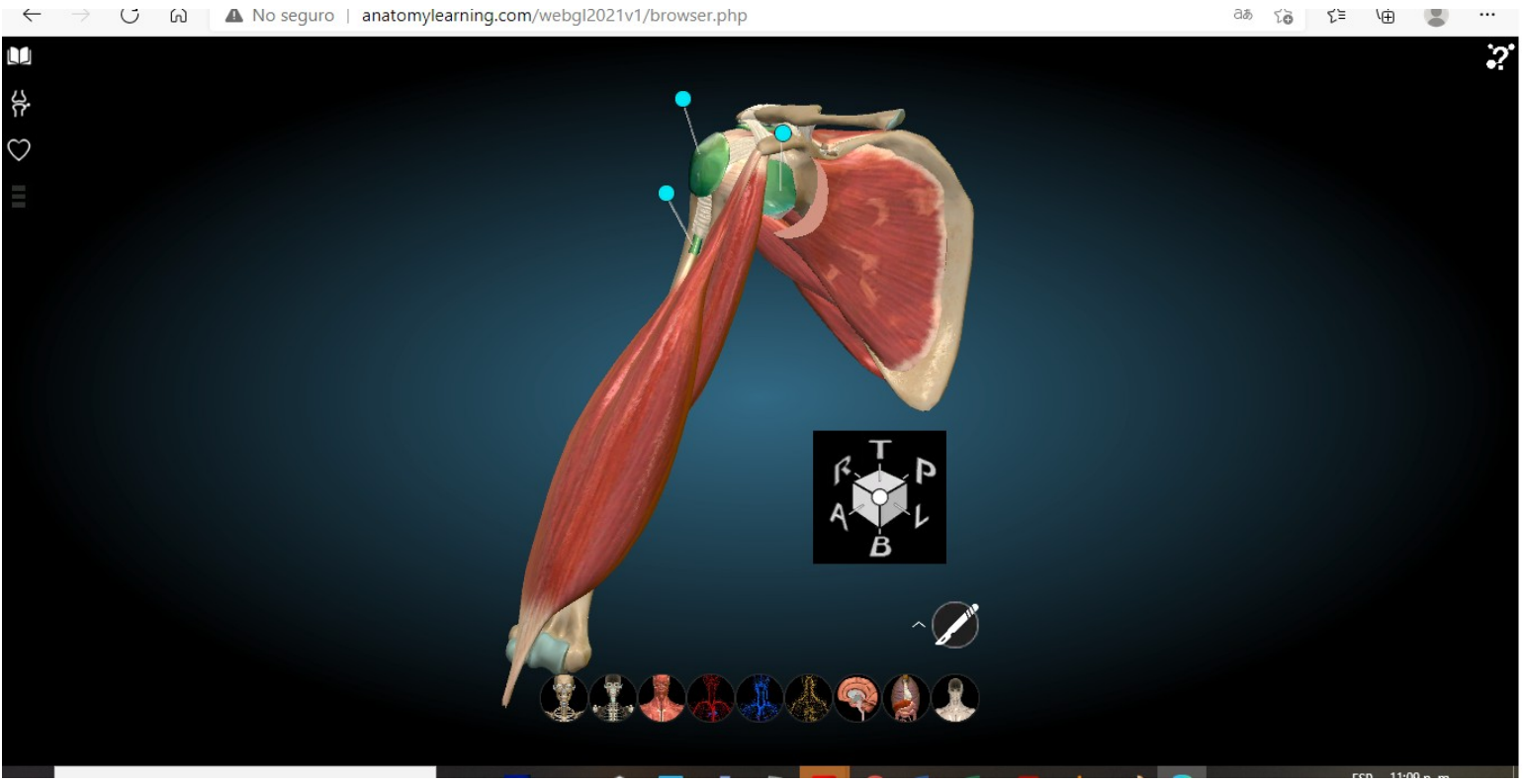

Figura 6. Cubo de vistas de Anatomy Learning 


\subsubsection{Anatomie3D}

Estas animaciones (Fig. 7) fueron desarrolladas por los servicios ICAP de la universidad Claude Bernard (Lyon I), para la enseñanza de anatomía funcional (23). Están publicadas en video en distintos idiomas, y en ellas se exhiben movimientos de rotación sobre un eje único. La referencia de orientación espacial es un avatar externo al objeto anatómico (arriba a la izquierda) que acompaña todas las rotaciones y movimientos del objeto.

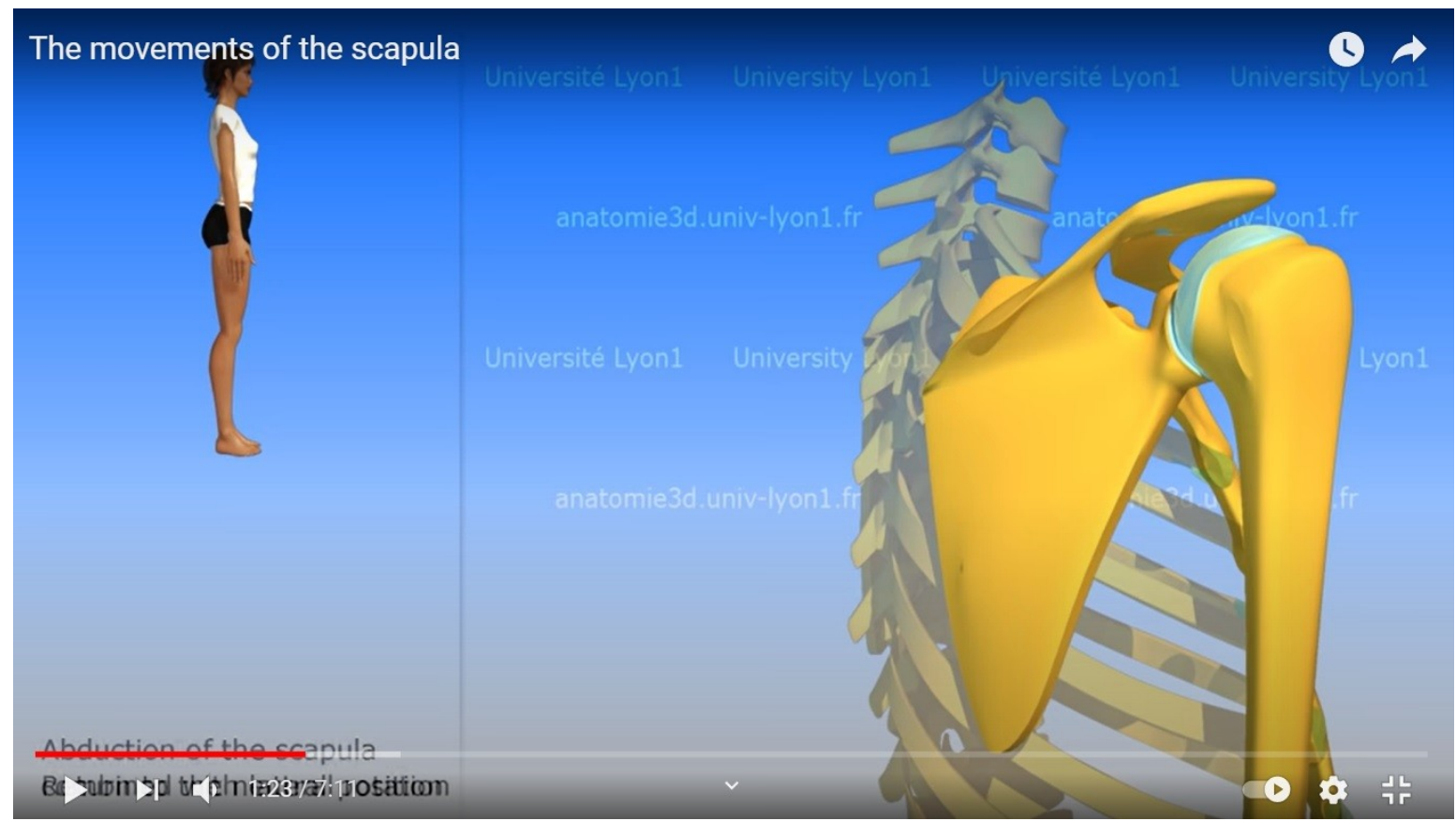

Figura 7. Anatomie3D. Animaciones de los movimientos de la escápula.

\section{Referencias}

2. Ackerman MJ. Accessing the Visible Human Project. D-Lib Magazine. 1995. http://www.dlib.org/dlib/october95/10ackerman.html

3. Hegarty M. Diagrams in the Mind and in the World: Relations between Internal and External Visualizations. En: Blackwell AF, Marriott K, Shimojima A, editores. Diagrammatic Representation and Inference. Berlin, Heidelberg: Springer; 2004. p. 1-13. (Lecture Notes in Computer Science). https://link.springer.com/chapter/10.1007/978-3-540-25931-2_1

4. Marr D, Nishihara HK. Representation and recognition of the spatial organization of three-dimensional shapes. Proc R Soc Lond B Biol Sci. 1978 Feb 23;200(1140):269-94. http://doi.org/10.1098/rspb.1978.0020

5. Jolicoeur, P. The time to name disoriented natural objects. Mem Cogn 13, 289-303 (1985). https://doi.org/10.3758/BF03202498

6. Jolicoeur P, Humphrey GK. Perception of rotated two-dimensional and three-dimensional objects and visual shapes. En: Perceptual constancy: Why things look as they do. New York, NY, US: Cambridge University Press; 1998. p. 69-123. https://psycnet.apa.org/record/1998-08068-003

7. Lawson R. Achieving visual object constancy across plane rotation and depth rotation. Acta Psychol (Amst). 1999 Sep;102(2-3):221-45. http://doi.org/10.1016/s0001-6918(98)00052-3

8. Lawson R, Humphreys GW, Jolicoeur P. The combined effects of plane disorientation and foreshortening on picture naming: one manipulation or two? J Exp Psychol Hum Percept Perform. 2000 Apr;26(2):568-81. http://dor.org/10.1037//0096-1523.26.2.568

9. Tarr MJ. Visual object recognition: Can a single mechanism suffice? En: Perception of faces, objects, and scenes: Analytic and holistic processes. New York, NY, US: Oxford University Press; 2003. p. 177-207. (Advances in visual cognition). https:/ / psycnet.apa.org/record/2003-88086-007

10. Graf M. Coordinate transformations in object recognition. Psychol Bull. 2006 Nov;132(6):920-45. http://doi.org/10.1037/0033-2909.132.6.920 
11. Bülthoff HH, Edelman SY, Tarr MJ. How are three-dimensional objects represented in the brain? Cereb Cortex. 1995 May-Jun;5(3):247-60. http://doi.org/10.1093/cercor/5.3.247.

12. Cutzu F, Edelman S. Canonical views in object representation and recognition. Vision Res. 1994 Nov;34(22):3037-56. http://doi.org/10.1016/0042-6989(94)90277-1

13. Edelman S, Bülthoff HH. Orientation dependence in the recognition of familiar and novel views of threedimensional objects. Vision Res. 1992 Dec;32(12):2385-400. http:/ / doi.org/10.1016/0042-6989(92)90102-o.

14. Diwadkar VA, McNamara TP. Viewpoint Dependence in Scene Recognition. Psychol Sci. 1 de julio de 1997;8(4):302-7. https://doi.org/10.1111/j.1467-9280.1997.tb00442.x

15. Tarr MJ. Rotating objects to recognize them: A case study on the role of viewpoint dependency in the recognition of three-dimensional objects. Psychon Bull Rev. 1995 Mar;2(1):55-82. http:/ / doi.org/10.3758/BF03214412.

16. Garsoffky B, Schwan S, Hesse FW. Viewpoint dependency in the recognition of dynamic scenes. J Exp Psychol Learn Mem Cogn. 2002 Nov;28(6):1035-50, PMID: 12450330.

17. Palmer, S., Rosch, E., and Chase, P. (1981). Canonical Perspective and the Perception of Objects. In J. Long, \& A. Baddeley (Eds.), International Symposium on Attention and Performance (Attention and performance IX) (pp. 135-151). Hillsdale, NJ: Lawrence Erlbaum Associates.

18. Hegarty M. Chapter 7 - Components of Spatial Intelligence. En: Psychology of Learning and Motivation [Internet]. Academic Press; 2010 [citado 5 de marzo de 2021]. p. 265-97. (The Psychology of Learning and
Motivation;
vol.
$52)$.
Disponible
en:

https://www.sciencedirect.com/science/article/pii/S0079742110520073

19. Keehner M, Hegarty M, Cohen C, Khooshabeh P, Montello DR. Spatial reasoning with external visualizations: what matters is what you see, not whether you interact. Cogn Sci. 2008 Oct;32(7):1099-132. http://doi.org/10.1080/03640210801898177

20. Hegarty M, Canham MS, Fabrikant SI. Thinking about the weather: How display salience and knowledge affect performance in a graphic inference task. J Exp Psychol Learn Mem Cogn. 2010 Jan;36(1):37-53. http://doi.org/10.1037/a0017683.

21. Levinson AJ, Weaver B, Garside S, McGinn H, Norman GR. Virtual reality and brain anatomy: a randomised trial of e-learning instructional designs. Med Educ. 2007 May;41(5):495501.http://doi.org/10.1111/j.1365-2929.2006.02694.x

22. Stull AT, Hegarty M, Mayer RE. Getting a handle on learning anatomy with interactive three-dimensional graphics. J Educ Psychol. 2009;101(4):803-16. http://doi.org/10.1037/a0016849

23. Halle M, Demeusy V, Kikinis R. The Open Anatomy Browser: A Collaborative Web-Based Viewer for Interoperable Anatomy Atlases. Front Neuroinformatics [Internet]. 2017 [citado 15 de marzo de 2021 ];11. Disponible en: https:/ / www.frontiersin.org/articles/10.3389/fninf.2017.00022/full

24. ICAP. Site anatomie 3D 2018 - Accueil [Internet]. 2018 [citado 8 de marzo de 2021]. Disponible en: http: / / anatomie3d.univ-lyon1.fr/webapp/website/website.html?id=3346735\&pageId=223660 